\title{
Correlation analysis of adjuvant chemotherapy duration and postoperative death in Chinese patients with colorectal cancer: a retrospective cohort study
}

\author{
Weiwei Chen ${ }^{1,2}$, Gang Wang 2 , Hongmin Dong², Wenling Wang ${ }^{2}$
}

${ }^{1}$ Department of Clinical Medicine, Guizhou Medical university, Guiyang, China 2Department of Abdominal Oncology, Affiliated Cancer Hospital of Guizhou Medical University, Guiyang, China

Submitted: 6 August 2020

Accepted: 19 December 2020

Arch Med Sci

DOI: https://doi.org/10.5114/aoms/131822

Copyright @ 2020 Termedia \& Banach

\section{Abstract}

Introduction: It is not known whether adjuvant chemotherapy duration is non-linearly associated with postoperative mortality risk. This study was designed to examine this relationship in Chinese patients with colorectal cancer.

Material and methods: This cohort study was conducted in 1171 participants with stage III and high-risk stage II colorectal cancer, who were treated in China between July 1, 2011, and September 1, 2018. The target independent variable was adjuvant chemotherapy duration, and the dependent variable was postoperative death.

Results: After adjusting for confounding factors, an increase in adjuvant chemotherapy duration was negatively correlated with the risk of postoperative death (hazard ratio $(\mathrm{HR})=0.95,95 \%$ confidence interval $(\mathrm{Cl})$ : 0.90-0.99). However, a non-linear relationship between therapy duration and postoperative death was observed in the group with bolus and infused fluorouracil with oxaliplatin (FOLFOX). In this group, the incidence of death decreased only when chemotherapy duration was more than 14 weeks (HR = $0.78,95 \% \mathrm{Cl}: 0.63-0.97)$. A stronger association between adjuvant chemotherapy duration and postoperative death was detected in the capecitabine and oxaliplatin (CAPOX) group ( $\mathrm{HR}=0.71,95 \% \mathrm{Cl}: 0.55-0.94)$ compared with the FOLFOX group ( $\mathrm{HR}=0.96,95 \% \mathrm{Cl}: 0.92-1.01)$.

Conclusions: The duration of adjuvant chemotherapy with the FOLFOX regimen is associated with a non-linear reduction in postoperative deaths, which is only apparent after at least 14 weeks of treatment. However, patients with colorectal cancer may receive CAPOX adjuvant treatment for a longer duration.

Key words: adjuvant chemotherapy duration, colorectal neoplasms, antineoplastic combined chemotherapy protocol, FOLFOX, CAPOX.

\section{Introduction}

Colorectal cancer is one of the five commonest causes of cancer deaths worldwide [1], and it is one of the major malignant tumours threatening health in China [2]. For several decades, the standard adjuvant treatment in patients diagnosed with stage III and high-risk stage II colorectal cancer has been a 6-month chemotherapy regimen with fluoropyrimidine and oxaliplatin $[3,4]$. The relationship between adjuvant chemotherapy

\author{
Corresponding author: \\ Weiwei Chen \\ Department \\ of Clinical Medicine \\ Guizhou Medical University \\ No. 1 Beijing Road \\ 550001 Guiyang \\ China \\ Phone: +860851 85893008 \\ Fax: +860851 85498530 \\ E-mail: weiweic043@163.com
}


duration and postoperative death is controversial [5]. Previous studies have shown that adjuvant chemotherapy consisting of 8 cycles or more is associated with a higher rate of survival [6]. However, a recent pooled analysis by the International Duration Evaluation of Adjuvant Therapy (IDEA) collaboration [7] shows that, in patients with stage III colon cancer, a shorter duration (3 months) of chemotherapy with capecitabine and oxaliplatin is as effective as treatment for 6 months. Due to differences in design, target population, chemotherapy regimen, and data analysis of the studies included in this analysis, additional studies are warranted. Thus, we were prompted to explore whether adjuvant chemotherapy duration is non-linearly associated with postoperative mortality and to identify the appropriate threshold of duration in a real-world study.

\section{Material and methods}

\section{Study design}

A retrospective cohort study was performed to assess the relationship between adjuvant chemotherapy duration and postoperative death. The target independent variable was adjuvant chemotherapy duration (continuous variable). The dependent variable was postoperative death (dichotomous variable: 1 = death; 0 = survival).

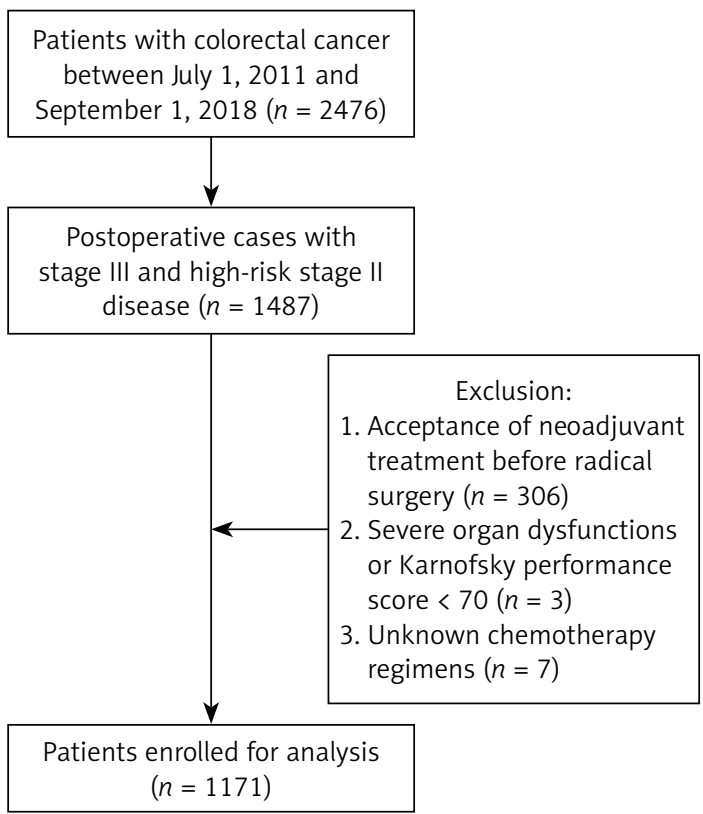

Figure 1. Flow chart of enrolment. A total of 2476 patients were diagnosed with colorectal cancer at our hospital between July 1, 2011, and September 1, 2018. Of these, 1487 patients had stage III or high-risk stage II disease and were treated with radical surgery. When cases with neoadjuvant treatment $(n=306)$, severe co-morbidity $(n=3)$, and unclear chemotherapy regimen $(n=7)$ were excluded, 1171 patients were deemed eligible for analysis

\section{Study population}

Data on participants with stage III and high-risk stage II colorectal cancer were non-selectively collected from the electronic medical records system of the Department of Abdominal Oncology, the Cancer Hospital of Guizhou Medical University, Guiyang, China. In order to safeguard patient privacy, identifiable participant data were not included. Because this was a retrospective cohort study, informed consent from participants was not required. The hospital's Ethics Review Committee approved the study.

Figure 1 shows the flow chart for enrolment. A total of 1171 participants who were treated at our hospital between July 1, 2011, and September 1, 2018 were enrolled. The inclusion criteria for this study were as follows: (1) a pathologically confirmed diagnosis of colonic or rectal adenocarcinoma, graded as either high-risk stage II disease (defined as tumour obstruction with or without tumour perforation preoperatively, poor differentiation, fewer than 12 lymph nodes detected, perineural infiltration, invasion of extramural venous or lymphatic vascular, or T4 disease) or stage III disease, (2) no neoadjuvant treatment, and (3) initial treatment by radical surgery. Exclusion criteria included: (1) unknown chemotherapy regimen, (2) severe heart, lung, liver, or kidney dysfunction unsuitable for adjuvant chemotherapy, and (3) a Karnofsky performance score (a measure of cancer patients' general well-being) of $<70$.

\section{Variables}

Adjuvant chemotherapy duration, defined as the time between the first cycle of chemotherapy initiated and the last cycle of chemotherapy accomplished, was measured as a continuous variable. The final outcome variable was postoperative death, which was dichotomous.

We listed in detail the covariates used in this study. Demographic factors were included as potential confounders, along with variables highlighted by previous studies examining factors associated with postoperative death or deemed important from our own clinical experience.

The final multivariable-adjusted models included age and pre-surgery levels of carcinoma antigen 19-9 (CA 19-9), carcinoembryonic antigen (CEA), prealbumin, prognosis nutritional index (PNI; serum albumin $+5 \times$ lymphocyte count), and the neutrophil-to-lymphocyte ratio (NLR) as continuous variables and gender, $\mathrm{T}$ stage, $\mathrm{N}$ stage, tumour differentiation, and chemotherapy regimen as categorical variables.

\section{Treatment protocol}

All participants had previously undergone radical surgery, which, for patients with rectal can- 
cer, consisted of total mesorectal excision. Based on published randomised controlled studies and the US National Comprehensive Cancer Network guidelines, the adjuvant chemotherapy regimens consisted of a combination of oxaliplatin and fluoropyrimidine. In the capecitabine and oxaliplatin (CAPOX) regimen, oxaliplatin was given every 3 weeks at a dose of $130 \mathrm{mg} / \mathrm{m}^{2}$ of body-surface area, and capecitabine was given twice daily for the first 2 weeks of every 3-week period. In the bolus and infused fluorouracil with oxaliplatin (FOLFOX) regimen, oxaliplatin was given every 2 weeks at a dose of $85 \mathrm{mg} / \mathrm{m}^{2}$. In addition, a 46-h infusion of fluorouracil was administered every 2 weeks [8-10].

\section{Follow-up procedure}

Participants were followed up through outpatient visits once every 3 months for the first 2 years, every 6 months for the next 3 years, and annually thereafter. Each follow-up visit included a complete physical examination and thoraco-abdominal and pelvic computed tomography scans. Serum levels of CEA and CA 19-9 were also assessed. Rectoscopy was carried out on an annual basis. The follow-up time began from the first clinical visit and continued until death occurred or until the last date of follow-up on June 1, 2019. Follow-up data were stored in the Cancer Hospital of Guizhou Medical University's electronic medical records system and were managed by the second author.

\section{Statistical analysis}

Continuous variables were expressed as mean \pm standard deviation and median (minimum, maximum) and categorical variables as a frequency or percentage. Student's $t$-test (normal distribution), $\chi^{2}$ test (categorical variables), or the Mann-Whitney $U$ test (skewed distribution) were applied to investigate differences in clinicopathological characteristics between tertiles of chemotherapy duration. Univariate and multivariate Cox proportional hazards models were employed to assess variables that may be associated with mortality. Three models were established: (1) unadjusted, (2) adjusted for gender, age, $\mathrm{T}$ stage, and $\mathrm{N}$ stage, and (3) adjusted for all the covariates presented in Table I. The Cox proportional hazards regression model with cubic spline functions and smooth curve fitting was adopted to address a non-linear relationship between adjuvant chemotherapy duration and postoperative death. If non-linearity was detected, the inflection point was calculated from a recursive algorithm, after which a 2 piecewise Cox proportional hazards model was established on either side of it. Ultimately, the most suitable model for fitting the association between the target independent variable and the outcome variable was determined by a log likelihood ratio test. Stratified Cox proportional hazards models were used to conduct subgroup analyses. After converting the target independent variable to a categorical variable by tertile, an interaction test was performed. Exploration of effect modification for these subgroup indicators was followed by a likelihood ratio test.

\section{Addressing missing data and sensitivity analysis}

Where covariate records were incomplete, dummy variables were used to indicate missing values. For each covariate, we assigned a missing record of 0 and additionally created a dummy variable $(0=$ not missing, $1=$ missing). Both variables were included in the model at the same time $(Y=$ $\mathrm{aX} 1+\mathrm{b} \times$ dummy variable).

We conducted a series of sensitivity analyses to guarantee the robustness of the data analysis. First, the adjuvant chemotherapy duration was converted into a categorical variable. The $p$-value for the trend was then calculated, in order to verify adjuvant chemotherapy duration as a continuous variable and observe the possibility of non-linearity. Second, due to the limitations of the Cox proportional hazards model in addressing non-linearity, a generalised additive model was used to adjust covariates; effect size was then compared with a fully adjusted model.

All data analysis was performed with the statistical software packages R (http://www.R-project. org, The R Foundation) and EmpowerStats (http:// www.empowerstats.com, X\&Y Solutions, Inc., Boston, MA, SUA). $P$-values $<0.05$ (2-sided) were considered statistically significant.

\section{Results}

\section{Baseline characteristics of enrolled participants}

A total of 1171 participants were enrolled into the final data analysis after screening (Figure 1). During follow-up, 118 deaths were observed $(118 / 1171=10.08 \%)$. Table I describes the baseline clinicopathological characteristics of enrolled patients across the tertiles of chemotherapy duration. With the exception of age, CEA, prealbumin, PNI, NLR, and chemotherapy regimen, no statistically significant differences were found between the groups for any of these measures. Compared to participants in the lower tertiles, participants in tertile 3 had higher prealbumin and PNI but were younger and had a lower CEA and NLR.

\section{Univariate analysis}

Table II shows the results of the univariate analysis. Using the univariate Cox proportional hazards model, gender, CA 19-9, T stage, and chemotherapy regimen were not associated with 
Table I. Characteristics of the study participants according to the duration of chemotherapy

\begin{tabular}{|c|c|c|c|c|}
\hline \multirow[t]{2}{*}{ Characteristics } & \multicolumn{3}{|c|}{ Duration of chemotherapy } & \multirow[t]{2}{*}{$P$-value } \\
\hline & $\begin{array}{c}\text { Tertile } 1 \\
(<8 \text { weeks })\end{array}$ & $\begin{array}{c}\text { Tertile } 2 \\
\text { (8-16 weeks) }\end{array}$ & $\begin{array}{c}\text { Tertile } 3 \\
(\geq 16 \text { weeks })\end{array}$ & \\
\hline No. of participants & 376 & 283 & 512 & \\
\hline $\begin{array}{l}\text { Age [years], mean (SD), } \\
\text { median (min.- max.) }\end{array}$ & $\begin{array}{c}65.64(13.08), \\
67.00(15.00-9.00)\end{array}$ & $\begin{array}{c}56.18(13.05), \\
59.00(18.00-79.00)\end{array}$ & $\begin{array}{c}55.35(11.73), \\
56.00(21.00-81.00)\end{array}$ & $<0.001$ \\
\hline Gender: & & & & 0.109 \\
\hline Male & $221(58.78 \%)$ & $162(57.24 \%)$ & $328(64.06 \%)$ & \\
\hline Female & $155(41.22 \%)$ & $121(42.76 \%)$ & $184(35.94 \%)$ & \\
\hline T stage: & & & & 0.309 \\
\hline $1-2$ & $23(6.12 \%)$ & $15(5.30 \%)$ & 20 (3.91\%) & \\
\hline $3-4$ & $353(93.88 \%)$ & $268(94.70 \%)$ & 492 (96.09\%) & \\
\hline N stage: & & & & 0.642 \\
\hline 0 & $183(48.67 \%)$ & $125(44.17 \%)$ & $253(49.41 \%)$ & \\
\hline 1 & $135(35.90 \%)$ & $113(39.93 \%)$ & $178(34.77 \%)$ & \\
\hline 2 & $58(15.43 \%)$ & 45 (15.90\%) & $81(15.82 \%)$ & \\
\hline Differentiation: & & & & 0.571 \\
\hline Poorly & $25(6.67 \%)$ & $22(7.77 \%)$ & $44(8.59 \%)$ & \\
\hline Moderate to high & 350 (93.33\%) & 261 (92.23\%) & 468 (91.41\%) & \\
\hline $\begin{array}{l}\text { CA199 [U/ml], mean (SD), } \\
\text { median (min.- max.) }\end{array}$ & $\begin{array}{c}49.99(467.84), \\
12.14(0.01-9028.00)\end{array}$ & $\begin{array}{c}22.06(47.48), \\
9.61(0.60-517.93)\end{array}$ & $\begin{array}{c}22.71(49.13), \\
10.25(0.00-700.00) \\
\end{array}$ & 0.264 \\
\hline $\begin{array}{l}\text { CEA [U/ml], mean (SD), } \\
\text { median (min.- max.) }\end{array}$ & $\begin{array}{c}9.53(19.45), 2.79 \\
(0.02-174.87) \\
\end{array}$ & $\begin{array}{l}9.38(17.35), 2.67 \\
(0.01-100.00)\end{array}$ & $\begin{array}{c}7.50(16.43), \\
2.30(0.00-159.70)\end{array}$ & 0.008 \\
\hline $\begin{array}{l}\text { Prealbumin [mg/l], mean } \\
\text { (SD), median (min.-max.) }\end{array}$ & $\begin{array}{c}189.43(61.60), \\
192.60(42.14-396.50)\end{array}$ & $\begin{array}{c}200.65(62.75), \\
200.38(24.84-446.00)\end{array}$ & $\begin{array}{c}205.61(59.96) \\
206.55(19.60-454.00)\end{array}$ & $<0.001$ \\
\hline $\begin{array}{l}\text { PNI, mean (SD), } \\
\text { median (min.-max.) }\end{array}$ & $\begin{array}{c}47.23(6.68), \\
47.63(25.40-67.30)\end{array}$ & $\begin{array}{c}48.89(5.94), \\
48.65(27.20-68.20)\end{array}$ & $\begin{array}{c}49.01(6.85), \\
48.90(23.50-133.60)\end{array}$ & $<0.001$ \\
\hline $\begin{array}{l}\text { NLR, mean (SD), } \\
\text { median (min.-max.) }\end{array}$ & $\begin{array}{c}4.16(11.40) \\
2.60(0.74-214.20)\end{array}$ & $\begin{array}{c}3.38(3.61) \\
2.36(0.72-35.46)\end{array}$ & $\begin{array}{c}3.36(3.59), \\
2.29(0.32-33.00)\end{array}$ & 0.027 \\
\hline Chemotherapy regimen: & & & & 0.022 \\
\hline FOLFOX & $81(80.20 \%)$ & $251(88.69 \%)$ & 418 (81.64\%) & \\
\hline CAPOX & $20(19.80 \%)$ & $32(11.31 \%)$ & $94(18.36 \%)$ & \\
\hline
\end{tabular}

CA - carcinoma antigen, CEA - carcinoembryonic antigen, PNI - prognostic nutritional index, NLR - neutrophil lymphocyte ratio, FOLFOX - fluorouracil, leucovorin, and oxaliplatin, CAPOX - capecitabine and oxaliplatin.

postoperative death. Compared to reference values, chemotherapy duration (hazard ratio $(\mathrm{HR})=$ 0.90, 95\% confidence interval $(\mathrm{Cl}): 0.87-0.93)$, levels of prealbumin ( $\mathrm{HR}=1.00,95 \% \mathrm{Cl}$ : 0.99-1.00) and PNI ( $\mathrm{HR}=0.95,95 \% \mathrm{Cl}: 0.92-0.97)$, and moderate to high differentiation $(\mathrm{HR}=0.49,95 \% \mathrm{Cl}$ : $0.30-0.81)$ were negatively associated with postoperative death. However, age $(\mathrm{HR}=1.02,95 \% \mathrm{Cl}$ : 1.01-1.04), levels of CEA (HR $=1.01,95 \% \mathrm{Cl}$ : $1.01-1.02)$, NLR (HR $=1.01,95 \% \mathrm{Cl}: 1.00-1.02)$, $\mathrm{N} 1$ stage $(\mathrm{HR}=2.04,95 \% \mathrm{Cl}: 1.26-3.29)$, and N2 stage $(\mathrm{HR}=5.55,95 \% \mathrm{Cl}: 3.45-8.90)$ were all positively correlated with postoperative death.

\section{Results of unadjusted and adjusted Cox proportional hazards models}

Three models were employed to determine whether there is an independent association between adjuvant chemotherapy duration and postoperative death (Table III). In the unadjusted model, the HR indicated the difference in adjuvant chemotherapy duration associated with an increased risk of postoperative death. Thus, the HR of 0.9 (95\% Cl: $0.87-0.93)$ estimated by this model indicated that each additional week of chemotherapy was associated with a 10\% decrease in mortality. Meanwhile, in the minimally adjusted 
Table II. Results of univariate analysis

\begin{tabular}{|c|c|c|}
\hline \multirow[t]{2}{*}{ Variables } & \multirow[t]{2}{*}{ Mean $\pm \mathrm{SD} / n(\%)$} & \multirow{2}{*}{$\begin{array}{l}\text { Postoperative death } \\
\text { HR }(95 \% \mathrm{Cl}), P \text {-value }\end{array}$} \\
\hline & & \\
\hline Duration of chemotherapy [week] & $8.94 \pm 7.29$ & $0.90(0.87,0.93),<0.0001$ \\
\hline \multicolumn{3}{|c|}{ Tertile of duration of chemotherapy [week]: } \\
\hline Tertile 1 & $376(32.11 \%)$ & 1.0 \\
\hline Tertile 2 & $283(24.17 \%)$ & $0.41(0.26,0.65), 0.0001$ \\
\hline Tertile 3 & $512(43.72 \%)$ & $0.21(0.13,0.33),<0.0001$ \\
\hline \multicolumn{3}{|l|}{ Gender: } \\
\hline Male & $711(60.72 \%)$ & 1.0 \\
\hline Female & $460(39.28 \%)$ & $1.32(0.92,1.89), 0.1364$ \\
\hline Age & $58.86 \pm 13.34$ & $1.02(1.01,1.04), 0.0011$ \\
\hline \multicolumn{3}{|l|}{ Age tertile: } \\
\hline Low & $390(33.30 \%)$ & 1.0 \\
\hline Middle & $390(33.30 \%)$ & 1.15 (0.70, 1.89), 0.5699 \\
\hline High & $391(33.39 \%)$ & $2.13(1.37,3.33), 0.0009$ \\
\hline \multicolumn{3}{|l|}{ T stage: } \\
\hline $1-2$ & $58(4.95 \%)$ & 1.0 \\
\hline $3-4$ & $1113(95.05 \%)$ & $1.29(0.53,3.17), 0.5747$ \\
\hline \multicolumn{3}{|l|}{ N stage: } \\
\hline 0 & $561(47.91 \%)$ & 1.0 \\
\hline 1 & $426(36.38 \%)$ & $2.04(1.26,3.29), 0.0036$ \\
\hline 2 & $184(15.71 \%)$ & $5.55(3.45,8.90),<0.0001$ \\
\hline \multicolumn{3}{|l|}{ Differentiation: } \\
\hline Poorly & $91(7.78 \%)$ & 1.0 \\
\hline Moderate to high & 1079 (92.22\%) & $0.49(0.30,0.81), 0.0052$ \\
\hline CEA & $8.60 \pm 17.68$ & $1.01(1.01,1.02),<0.0001$ \\
\hline \multicolumn{3}{|l|}{ CEA tertile: } \\
\hline Low & $388(33.16 \%)$ & 1.0 \\
\hline Middle & $392(33.50 \%)$ & $1.19(0.74,1.90), 0.4783$ \\
\hline High & $390(33.33 \%)$ & $1.63(1.05,2.54), 0.0312$ \\
\hline CA199 & $31.36 \pm 268.84$ & 1.00 (1.00, 1.00), 0.6799 \\
\hline \multicolumn{3}{|l|}{ CA199 tertile: } \\
\hline Low & $388(33.30 \%)$ & 1.0 \\
\hline Middle & $388(33.30 \%)$ & $1.02(0.62,1.69), 0.9312$ \\
\hline High & 389 (33.39\%) & 2.03 (1.31, 3.16), 0.0016 \\
\hline \multicolumn{3}{|l|}{ Chemotherapy regimen: } \\
\hline FOLFOX & $750(83.71 \%)$ & 1.0 \\
\hline CAPOX & $146(16.29 \%)$ & $0.78(0.37,1.63), 0.5064$ \\
\hline Prealbumin & $199.22 \pm 61.52$ & $1.00(0.99,1.00), 0.0018$ \\
\hline \multicolumn{3}{|l|}{ Prealbumin tertile: } \\
\hline Low & $390(33.30 \%)$ & 1.0 \\
\hline Middle & $386(32.96 \%)$ & $0.57(0.37,0.88), 0.0106$ \\
\hline High & 395 (33.73\%) & $0.52(0.34,0.81), 0.0038$ \\
\hline
\end{tabular}


Table II. Cont.

\begin{tabular}{|c|c|c|}
\hline \multirow[t]{2}{*}{ Variables } & \multirow[t]{2}{*}{ Mean $\pm \mathrm{SD} / n$ (\%) } & Postoperative death \\
\hline & & HR ( $95 \% \mathrm{Cl}), P$-value \\
\hline PNI & $48.41 \pm 6.63$ & $0.95(0.92,0.97), 0.0001$ \\
\hline \multicolumn{3}{|l|}{ PNI tertile: } \\
\hline Low & $390(33.30 \%)$ & 1.0 \\
\hline Middle & $390(33.30 \%)$ & $0.87(0.58,1.29), 0.4808$ \\
\hline High & 391 (33.39\%) & $0.45(0.27,0.74), 0.0016$ \\
\hline NLR & $3.62 \pm 7.11$ & 1.01 (1.00, 1.02), 0.0083 \\
\hline \multicolumn{3}{|l|}{ NLR tertile: } \\
\hline Low & 389 (33.22\%) & 1.0 \\
\hline Middle & $390(33.30 \%)$ & $0.93(0.58,1.49), 0.7636$ \\
\hline High & 392 (33.48\%) & $1.33(0.86,2.05), 0.1968$ \\
\hline
\end{tabular}

$H R$ - hazard ratio, CI - confidence interval, CA - carcinoma antigen, CEA - carcinoembryonic antigen, PNI - prognostic nutritional index, NLR - neutrophil lymphocyte ratio, FOLFOX - fluorouracil, leucovorin, and oxaliplatin, CAPOX - capecitabine and oxaliplatin.

model, an increase in adjuvant chemotherapy duration of 1 week resulted in a $9 \%$ decrease in mortality $(\mathrm{HR}=0.91,95 \% \mathrm{Cl}: 0.88-0.94)$. In the fully adjusted model, each additional week of chemotherapy decreased the risk of mortality by $5 \%$ ( $\mathrm{HR}=0.95,95 \% \mathrm{Cl}: 0.90-0.99)$. In order to conduct a sensitivity analysis, the adjuvant chemotherapy duration was converted from a continuous variable into tertiles. In the fully adjusted model, the $p$-value for the trend as a categorical variable was consistent, with the result as a continuous variable. Furthermore, the trend for the effect size among the tertiles was non-equidistant in this model.

\section{Results of non-linearity of adjuvant chemotherapy duration and postoperative death using the FOLFOX regimen}

After adjusting for age, CA 19-9, CEA, prealbumin, PNI, NLR, gender, $T$ stage, N stage, and differentiation, smooth curve analysis and the Cox proportional hazards regression model with cubic spline functions indicated that the relationship between adjuvant chemotherapy duration and postoperative death was non-linear in the FOLFOX group (Figure 2 and Table IV).

Because the $p$-value for the log likelihood ratio test was $<0.05$ in this group, the 2 piecewise Cox proportional hazards model was selected as the best fit for the association between adjuvant chemotherapy duration and postoperative death. The inflection point was calculated as 14 . On the right side of the inflection point, the effect value and $95 \% \mathrm{Cl}$ were 0.78 and $0.63-0.97$, respectively. On the left side, the effect value and $95 \% \mathrm{Cl}$ were 1.03 and $0.95-1.11$, respectively.

\section{Subgroup analysis}

Trends in HRs among different subgroups, stratified by chemotherapy regimen, are shown in Figure 3. Significant $(p<0.05)$ interactions between

Table III. Results of multivariable analysis

\begin{tabular}{|lccc|}
\hline Variable & \multicolumn{1}{c}{ Non-adjusted } & Adjust I & Adjust II \\
\cline { 2 - 4 } & HR $(\mathbf{9 5 \% ~ C l ) , P \text { -value }}$ & HR $(95 \% \mathrm{Cl}), P$-value & HR $(95 \% \mathrm{Cl}), P$-value \\
\hline $\begin{array}{l}\text { Duration of } \\
\text { chemotherapy }\end{array}$ & $0.90(0.87,0.93),<0.0001$ & $0.91(0.88,0.94),<0.0001$ & $0.95(0.90,0.99), 0.0126$ \\
\hline $\begin{array}{l}\text { Tertile of duration of } \\
\text { chemotherapy [week]: }\end{array}$ & 1.0 & & \\
\hline $\begin{array}{l}\text { Tertile 1 } \\
\text { Tertile 2 }\end{array}$ & $0.41(0.26,0.65), 0.0001$ & $0.48(0.29,0.79), 0.0039$ & $0.82(0.38,1.78), 0.6181$ \\
\hline Tertile 3 & $0.21(0.13,0.33),<0.0001$ & $0.25(0.15,0.42),<0.0001$ & $0.49(0.23,1.06), 0.0700$ \\
\hline$P$ for trend & $<0.00001$ & $<0.00001$ & 0.03597 \\
\hline
\end{tabular}

Non-adjusted model adjusted for: None. Adjust I adjust for: Gender; Age; N stage; T stage. Adjust II adjust for: Gender; Age; N stage; $T$ stage; Differentiation; Prealbumin; CEA; CA199; PNI; NLR; Chemotherapy regimen. HR - hazard ratio, CI - confidence interval, $C A$ - carcinoma antigen, CEA - carcinoembryonic antigen, PNI - prognostic nutritional index, NLR - neutrophil lymphocyte ratio. 
different chemotherapy regimens were assessed (Table V). In this study, a stronger association was detected in the CAPOX group $(\mathrm{HR}=0.71,95 \% \mathrm{Cl}$ : $0.55-0.94, p=0.0144)$ compared with the FOLFOX group $(\mathrm{HR}=0.96,95 \% \mathrm{Cl}: 0.92-1.01, p=0.0934)$.

\section{Discussion}

This retrospective cohort study explored the association between adjuvant chemotherapy duration and postoperative death in Chinese patients with stage III and high-risk stage II colorectal cancer. Overall, adjuvant chemotherapy duration was negatively associated with mortality. Age and levels of CEA, prealbumin, PNI, and NLR differed significantly with chemotherapy duration. Because this real-world study was not randomised, the general condition of the patients enrolled was inevitably different, and this may have had an effect on overall survival and chemotherapy dose. Therefore, the results were adjusted for these confounding factors.

Even though there are few data available on adjuvant chemotherapy for rectal cancer, patients with rectal cancer were included. Neoadjuvant chemoradiotherapy is the standard treatment in the majority of stage III rectal cancer patients. However, many surgeons in China prefer surgical treatment as the first intervention for patients with locally advanced colorectal cancer without cT4b. Therefore, this study included 377 stage III rectal cancer patients without neoadjuvant chemoradiotherapy. Study participants with rectal cancer had undergone radical surgery consisting of total mesorectal excision and had not received any preoperative chemotherapy, in accordance with the Scleroderma: Cyclophosphamide Or Transplantation (SCOT) study [11]. The current study did not reveal different effects of adjuvant chemotherapy duration between patients with colon or rectal cancer, reflecting the results of the SCOT study [11]. Therefore, there is no reason to believe that rectal cancer patients should be treated differently from the entire population in clinical practice. The current study included both stage III

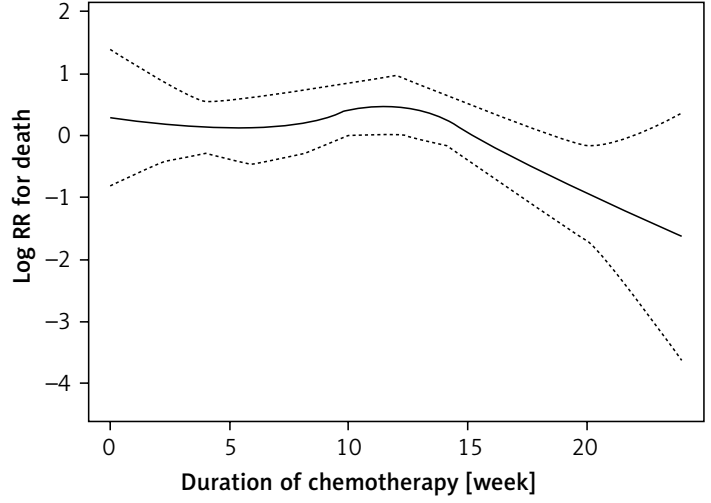

Figure 2. The curved line shows the relationship between adjuvant chemotherapy duration and mortality risk in the FOLFOX group. After adjusting for age, CA 19-9, CEA, prealbumin, PNI, NLR, gender, $\mathrm{T}$ stage, $\mathrm{N}$ stage, and differentiation, a non-linear relationship is observed. The area between the 2 dotted lines is expressed as a $95 \% \mathrm{Cl}$. Each point shows the length of adjuvant chemotherapy duration and is connected to form a continuous line. Below 14 weeks' duration, the length of adjuvant chemotherapy is not correlated with a decrease in mortality. Conversely, there is a significantly negative correlation with mortality at durations of $\geq 14$ weeks

and high-risk stage II patients. The majority of the stage II patients enrolled in this study showed microsatellite stability and presented with the same high-risk factors as seen in the IDEA study. Both stage II and III patients benefited from adjuvant chemotherapy in the current study. Future research should focus on whether the number and nature of high-risk factors and mutation of the BRAF gene modify the association between adjuvant chemotherapy duration and postoperative death.

Because previous studies have suggested that the optimal duration of adjuvant chemotherapy may vary according to treatment regimen [7, 12], we also conducted a subgroup analysis, stratified by this factor. This showed that the chemotherapy regimen modifies the association between adjuvant chemotherapy duration and postoperative death, because a significantly stronger as-

Table IV. Results of the two-piecewise linear regression model regarding the different chemotherapy regimens

\begin{tabular}{|lcc|}
\hline Chemotherapy regimen & FOLFOX & CAPOX \\
\cline { 2 - 3 } & HR $(95 \% \mathrm{Cl}), P$-value & HR $(95 \% \mathrm{Cl}), P$-value \\
\hline Inflection point of duration of chemotherapy: & & \\
\hline$<14$ & $1.03(0.95,1.11), 0.4824$ & $0.65(0.39,1.09), 0.1035$ \\
\hline$\geq 14$ & $0.78(0.63,0.97), 0.0250$ & $1.05(0.47,2.33), 0.9125$ \\
\hline$P$ for log likelihood ratio test & 0.017 & 0.464 \\
\hline
\end{tabular}

Adjust for: gender; age; N stage; T stage; differentiation; Prealbumin; CEA; CA199; PNI; NLR. HR - hazard ratio, CI-confidence interval, FOLFOX - fluorouracil, leucovorin, and oxaliplatin, CAPOX - capecitabine and oxaliplatin, CA - carcinoma antigen, CEA - carcinoembryonic antigen, PNI - prognostic nutritional index, NLR - neutrophil lymphocyte ratio. 


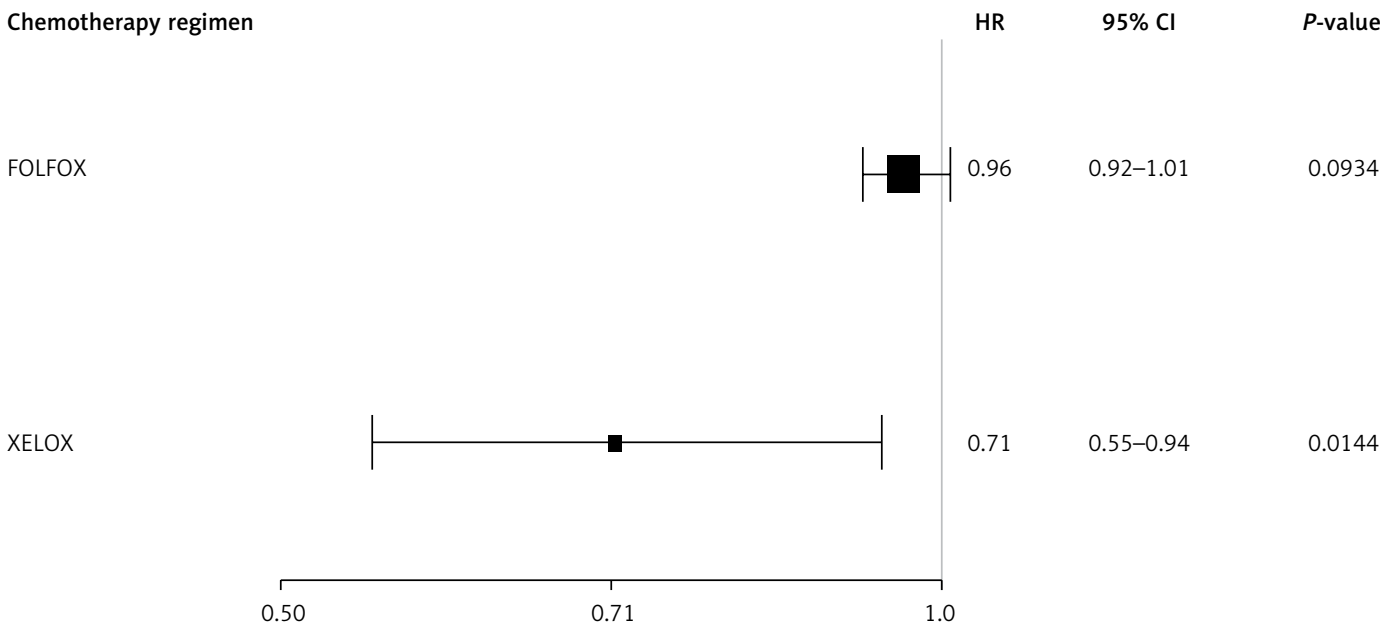

Figure 3. Analysis of the association, stratified by chemotherapy regimen, between adjuvant chemotherapy duration and postoperative death in Chinese patients with stage III and high-risk stage II colorectal cancer. This analysis is adjusted for gender, age, N stage, T stage, differentiation, prealbumin, CEA, CA 19-9, PNI, and NLR. HR - hazard ratio

Table V. Analysis of interactions between various chemotherapy regimens and duration of adjuvant chemotherapy

\begin{tabular}{|llcc|}
\hline Model & \multicolumn{1}{c}{ FOLFOX } & CAPOX & $P$ interaction \\
\cline { 2 - 4 } & HR $(95 \% \mathrm{Cl}), \boldsymbol{P}$-value & HR $(95 \% \mathrm{Cl}), \boldsymbol{P}$-value & 0.1111 \\
\hline Crude & $0.94(0.90,0.99), 0.0122$ & $0.85(0.74,0.97), 0.0134$ & 0.1079 \\
\hline Model I & $0.95(0.91,0.99), 0.0283$ & $0.85(0.75,0.97), 0.0185$ & 0.0053 \\
\hline Model II & $0.96(0.92,1.01), 0.0934$ & $0.71(0.55,0.94), 0.0144$ & 0 \\
\hline
\end{tabular}

Model I adjusted for: gender; age; N stage; T stage. Model II adjusted for: gender; age; N stage; T stage; differentiation; Prealbumin; CEA; CA199; PNI; NLR. HR - hazard ratio, CI - confidence interval, FOLFOX - fluorouracil, leucovorin, and oxaliplatin, CAPOX - capecitabine and oxaliplatin, CA - carcinoma antigen, CEA - carcinoembryonic antigen, PNI - prognostic nutritional index, NLR - neutrophil lymphocyte ratio.

sociation was observed in the CAPOX group compared with the FOLFOX group. In this latter group, non-linearity assessment suggested that a negative association was only observed when adjuvant chemotherapy was used for more than 14 weeks. This may be due to the threshold effect. These results are consistent with the findings from previous studies, which suggest that patients following the FOLFOX regimen may benefit from adjuvant chemotherapy lasting more than 3 months [7].

However, some previous findings are inconsistent with the current study. Grothey et al. [7] report that for patients treated with the CAPOX regimen, treatment durations of 3 and 6 months are equally effective. However, in our real-world analysis of Chinese patients, the mortality rate in this treatment group decreased with each additional week of adjuvant chemotherapy. The discordance between our results and those of previous studies is probably due to the following factors: first, the research population was different. The majority of studies reporting findings inconsistent with our own include patients from Europe and America. There are ethnic differences in oral fluoropyrimidine metabolism, with the threshold in East Asian populations being higher compared with Caucasians [13]. Therefore, longer durations of the CAPOX adjuvant regimen might be beneficial in Chinese patients. Second, compared with our studies, previous investigations did not take into account the effects of tumour differentiation or markers, or indices of nutrition (prealbumin and $\mathrm{PNI}$ ) and inflammation (NLR), on the association between adjuvant chemotherapy duration and postoperative death when adjusting for covariates. However, previous studies have confirmed that these variables are related to colorectal cancer prognosis [14-23].

Previous studies suggest that different adjuvant chemotherapy regimens will have different optimal durations [7, 11, 12], and this is confirmed by the current study. However, because it was not a randomised study, it is not possible to state clearly which regimen is superior. The findings also suggest that modifications in chemotherapy regimens may influence their efficacy, because the CAPOX group experienced lower mortality. Loree et al. [24] reported a cohort study showing that, compared with the FOLFOX regimen, CAPOX is associated with improved disease-free surviv- 


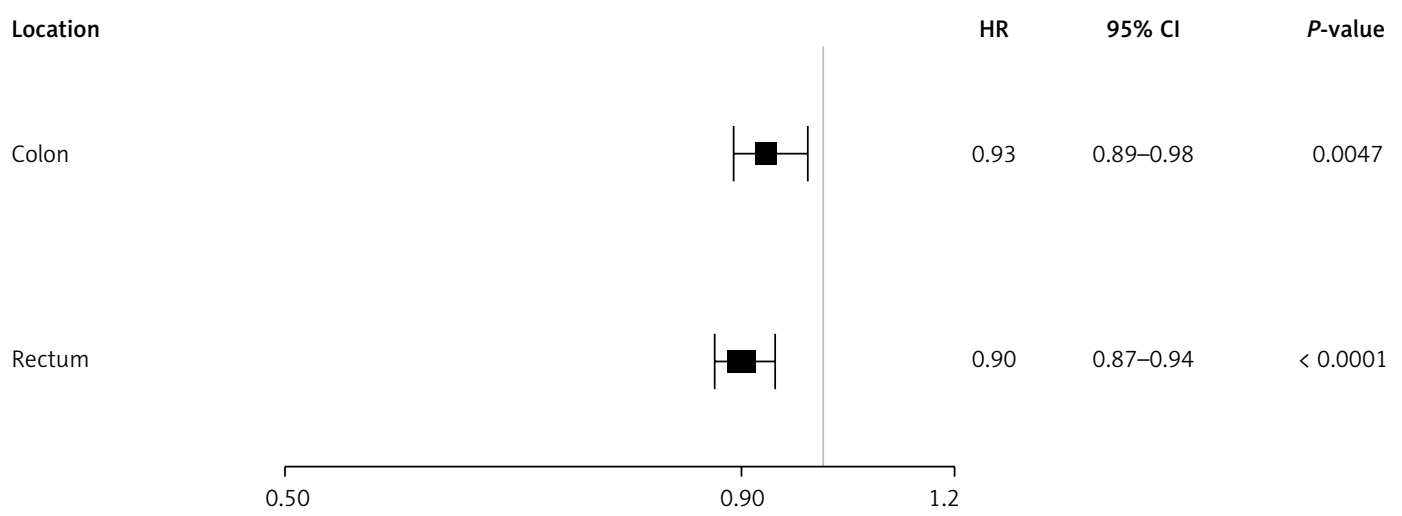

Figure 4. Analysis of the association, stratified by tumour location, between adjuvant chemotherapy duration and postoperative death in Chinese patients with stage III and high-risk stage II colorectal cancer. This analysis is adjusted for gender, age, N stage, T stage, differentiation, prealbumin, CEA, CA 19-9, PNI, and NLR. HR - hazard ratio

Table VI. Analysis of interactions between tumour location and duration of adjuvant chemotherapy

\begin{tabular}{|lccc|}
\hline Model & \multicolumn{1}{c}{ Colon } & Rectum & $P$ interaction \\
\cline { 2 - 3 } & HR $(95 \% \mathrm{Cl}), \boldsymbol{P}$-value & HR $(95 \% \mathrm{Cl}), \boldsymbol{P}$-value & \\
\hline Crude & $0.91(0.87,0.95),<0.0001$ & $0.89(0.86,0.93),<0.0001$ & 0.6551 \\
\hline Model I & $0.92(0.88,0.97), 0.0009$ & $0.90(0.86,0.94),<0.0001$ & 0.3506 \\
\hline Model II & $0.93(0.89,0.98), 0.0047$ & $0.90(0.87,0.94),<0.0001$ & 0.2850 \\
\hline
\end{tabular}

Model I adjusted for: gender; age; $N$ stage; T stage. Model II adjusted for: gender; age; N stage; T stage; differentiation; Prealbumin; CEA; CA199; PNI; NLR. HR - hazard ratio, CI - confidence interval, CA - carcinoma antigen, CEA - carcinoembryonic antigen, PNI-prognostic nutritional index, NLR - neutrophil lymphocyte ratio.

al. In addition, Chang [6] reported that more than 8 cycles of CAPOX treatment reduces the risk of death in locoregionally advanced rectal cancer in Chinese patients. This is consistent with our findings from subgroup analyses and implies that the regimen selection process should take the patient's ethnicity into consideration.

Our study has several strengths. Non-linearity and the threshold effect in the FOLFOX treatment group were addressed and explored further. Strict statistical adjustment was applied to minimise residual confounders in this study. Target independent variables were treated as both categorical and continuous variables. This approach increased the robustness of results. Finally, effect modifier factor analysis made better use of data and yielded stable conclusions in different subgroups. The results of this study should add to future research into predictive models of postoperative death.

However, this study has 2 limitations. First, patients with neoadjuvant treatment were excluded from this study; thus, the findings are not relevant to these patients. Second, the sample size of the CAPOX group is relatively small compared with that of the FOLFOX group. To confirm our conclusions, future studies need to enrol more patients into the CAPOX protocol.

In conclusion, overall, adjuvant chemotherapy duration is negatively associated with mortality in
Chinese patients with colorectal cancer. However, the relationship varies with chemotherapy regimen. In the FOLFOX group, adjuvant chemotherapy duration is associated with lower mortality in a non-linear relationship, with an effect only being seen if treatment lasts for more than 14 weeks. Compared with the FOLFOX group, the CAPOX group show a stronger negative correlation with mortality risk. Therefore, a longer duration of adjuvant chemotherapy with the CAPOX regimen may be appropriate for Chinese patients with colorectal cancer.

\section{Acknowledgments}

This work was supported by the Guiyang Medical College Doctoral Research Fund (YJ2015-380), Guizhou Education Department Youth Science and Technology Project (PT2016-58), Guizhou Science and Technology Plan Project ([2018]5779-33), and Guizhou Provincial Department of Education Innovation Group Research Project (KY[2018]020).

\section{Conflict of interest}

The authors declare no conflict of interest.

\section{References}

1. Bray F, Ferlay J, Soerjomataram I, Siegel RL, Torre LA, Jemal A. Global cancer statistics 2018: GLOBOCAN estimates of incidence and mortality worldwide for 
36 cancers in 185 countries. CA Cancer J Clin 2018; 68: 394-424.

2. Chen WQ, Li H, Sun KX, et al. Report of cancer incidence and mortality in China, 2014. Chin J Oncol 2018; 40: 5-13.

3. André T, Boni C, Navarro M, et al. Improved overall survival with oxaliplatin, fluorouracil, and leucovorin as adjuvant treatment in stage II or III colon cancer in the MOSAIC trial. J Clin Oncol 2009; 27: 3109-16.

4. Kuebler JP, Wieand HS, O'Connell MJ, et al. Oxaliplatin combined with weekly bolus fluorouracil and leucovorin as surgical adjuvant chemotherapy for stage II and III colon cancer: results from NSABP C-07. J Clin Oncol 2007; 25: 2198-204.

5. Kumar A, Peixoto RD, Kennecke HF, et al. Effect of adjuvant FOLFOX chemotherapy duration on outcomes of patients with stage III colon cancer. Clin Colorectal Cancer 2015; 14: 262-8.

6. Chang H, Yu X, Chen K, et al. Prognostic value of the cycle number of perioperative chemotherapy in locoregionally advanced rectal cancer: a propensity score matching analysis. J Cancer 2018; 9: 4346-54.

7. Grothey A, Sobrero AF, Shields AF, et al. Duration of adjuvant chemotherapy for stage III colon cancer. N Engl J Med 2018; 378: 1177-88.

8. Haller DG, Tabernero J, Maroun J, et al. Capecitabine plus oxaliplatin compared with fluorouracil and folinic acid as adjuvant therapy for stage III colon cancer. J Clin Oncol 2011; 29: 1465-71.

9. André T, Boni C, Mounedji-Boudiaf L, et al.; Multicenter International Study of Oxaliplatin/5-Fluorouracil/Leucovorin in the Adjuvant Treatment of Colon Cancer (MOSAIC) Investigators. Oxaliplatin, fluorouracil, and leucovorin as adjuvant treatment for colon cancer. N Engl J Med 2004; 350: 2343-51.

10. Benson AB, Venook AP, Al-Hawary MM, et al. NCCN Guidelines Insights: Colon Cancer, Version 2.2018. J Natl Compr Canc Netw 2018; 16: 359-69.

11. Iveson TJ, Kerr RS, Saunders MP, et al. 3 versus 6 months of adjuvant oxaliplatin-fluoropyrimidine combination therapy for colorectal cancer (SCOT): an international, randomised, phase 3, non-inferiority trial. Lancet Oncol 2018; 19: 562-78.

12. Souglakos J, Boukovinas I, Kakolyris S, et al. Three versus six months adjuvant FOLFOX or CAPOX for high risk stage II and stage III colon cancer patients: the efficacy results of Hellenic Oncology Research Group (HORG) participation to the International Duration Evaluation of Adjuvant chemotherapy (IDEA) project. Ann Oncol 2019; 30: 1304-10.

13. Haller DG, Cassidy J, Clarke SJ, et al. Potential regional differences for the tolerability profiles of fluoropyrimidines. J Clin Oncol 2008; 26: 2118-23.

14. Mori T, Hirota T, Ohashi Y, Kodaira S. Significance of histologic type of primary lesion and metastatic lymph nodes as a prognostic factor in stage III colon cancer. Dis Colon Rectum 2006; 49: 982-92.

15. Duffy MJ. Carcinoembryonic antigen as a marker for colorectal cancer: is it clinically useful? Clin Chem 2001; 47: 624-30.

16. Forones NM, Tanaka M. CEA and CA 19-9 as prognostic indexes in colorectal cancer. Hepatogastroenterology 1999; 46: 905-8.

17. Lai CC, You JF, Yeh CY, et al. Low preoperative serum albumin in colon cancer: a risk factor for poor outcome. Int J Colorectal Dis 2011; 26: 473-81.
18. Shibutani M, Maeda K, Nagahara H, et al. The prognostic significance of the postoperative prognostic nutritional index in patients with colorectal cancer. BMC Cancer 2015; 15: 521

19. Li MX, Liu XM, Zhang XF, et al. Prognostic role of neutrophil-to-lymphocyte ratio in colorectal cancer: a systematic review and meta-analysis. Int J Cancer 2014; 134: 2403-13.

20. Casadei-Gardini A, Scarpi E, Ulivi P, et al. Prognostic role of a new inflammatory index with neutrophil-to-lymphocyte ratio and lactate dehydrogenase (CII: Colon Inflammatory Index) in patients with metastatic colorectal cancer: results from the randomized Italian Trial in Advanced Colorectal Cancer (ITACa) study. Cancer Manag Res 2019; 11: 4357-69.

21. Inamoto S, Kawada K, Okamura R, Hida K, Sakai Y. Prognostic impact of the combination of neutrophil-to-lymphocyte ratio and Glasgow prognostic score in colorectal cancer: a retrospective cohort study. Int J Colorectal Dis 2019; 34: 1303-15.

22. Zhan X, Sun X, Hong Y, Wang Y, Ding K. Combined detection of preoperative neutrophil-to-lymphocyte ratio and CEA as an independent prognostic factor in nonmetastatic patients undergoing colorectal cancer resection is superior to NLR or CEA alone. Biomed Res Int 2017; 2017: 3809464

23. Zhao J, Xu J, Zhang R. Clinical and prognostic significance of pathological and inflammatory markers in mucinous rectal cancer patients receiving neoadjuvant chemoradiotherapy and curative surgery. Med Sci Monit 2017; 23: 4826-33.

24. Loree JM, Sha A, Soleimani M, et al. Survival impact of CAPOX Versus FOLFOX in the adjuvant treatment of stage III colon cancer. Clin Colorectal Cancer 2018; 17: 156-63 